\title{
The SSDF rating list, 2020-07, in memoriam Tony Hedlund
}

Article

Accepted Version

SSDF_2020-07_ICGA_J report

Sandin, L. and Haworth, G. (2020) The SSDF rating list, 202007, in memoriam Tony Hedlund. ICGA Journal, 42 (2-3). p. 242. ISSN 1389-6911 doi: https://doi.org/10.3233//CG-200155 Available at https://centaur.reading.ac.uk/91758/

It is advisable to refer to the publisher's version if you intend to cite from the work. See Guidance on citing.

Published version at: https://content.iospress.com/articles/icga-journal/icg200155

To link to this article DOI: http://dx.doi.org/10.3233/ICG-200155

Publisher: The International Computer Games Association

All outputs in CentAUR are protected by Intellectual Property Rights law, including copyright law. Copyright and IPR is retained by the creators or other copyright holders. Terms and conditions for use of this material are defined in the End User Agreement.

\section{www.reading.ac.uk/centaur}

\section{CentAUR}

Central Archive at the University of Reading

Reading's research outputs online 


\title{
The SSDF Rating List, 2020-07, in memoriam Tony Hedlund
}

\author{
Lars Sandin and Guy Haworth ${ }^{1}$ \\ Chairman, Svenska schackdatorföreningen; Reading, England
}

\begin{tabular}{|c|c|c|c|c|c|c|}
\hline \# Name & Rating & $+1-$ & Games & Win \% & Against & Notes \\
\hline 01 Stockfish 11 x64 1800X $3.6 \mathrm{GHz}$ & 3558 & $48 / 42$ & 330 & $76 \%$ & 3354 & NE; A, Tord Romstad, Marco Costalba \& Joona Kiiski \\
\hline 03 Stockfish 9 x64 1800X $3.6 \mathrm{GHz}$ & 3484 & $27 / 25$ & 842 & $71 \%$ & 3325 & 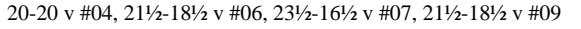 \\
\hline 06 Komodo 12.3 x64 1800X $3.6 \mathrm{GHz}$ & 3454 & $27 / 26$ & 760 & $66 \%$ & 3334 & $20-20 \mathrm{v} \# 07,22^{1} / 2-171 / 2 \mathrm{v} \# 09,20-20 \mathrm{v} \# 11,22-18 \mathrm{v} \# 13$ \\
\hline 07 Stockfish 9 x64 Q6600 2.4 GHz & 3444 & $32 / 31$ & 480 & $56 \%$ & 3401 & $16^{1 / 2-231 / 2} \mathrm{v} \# 03,20-20 \mathrm{v} \# 08,22-18 \mathrm{v} \# 12,21 \frac{1}{1} 2-181 / 2 \mathrm{v} \# 13$ \\
\hline 08 Komodo 12.3 x64 Q6600 $2.4 \mathrm{GHz}$ & 3436 & $38 / 36$ & 360 & $60 \%$ & 3361 & 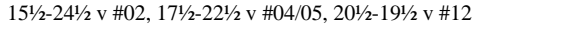 \\
\hline 11 Stockfish 8 x64 Q6600 $2.4 \mathrm{GHz}$ & 3411 & $31 / 30$ & 560 & $65 \%$ & 3301 & 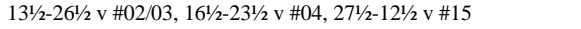 \\
\hline 12 Komodo 13.02 MCTS x64 1800X $3.6 \mathrm{GHz}$ & 3401 & $31 / 30$ & 520 & $57 \%$ & 3346 & 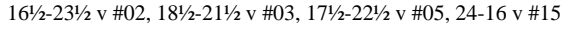 \\
\hline 13 Komodo 11.01 x64 1800X $3.6 \mathrm{GHz}$ & 3397 & $20 / 19$ & 394 & $69 \%$ & 3259 & 9-31 v \#01, 111/2-281/2 v \#02, 15-25 v \#03, 18-22 v \#04 \\
\hline 14 Deep Shredder 13 x64 1800X $3.6 \mathrm{GHz}$ & 3357 & $24 / 24$ & 880 & $64 \%$ & 3256 & A, Stefan Meyer-Kahlen; 13-27 v \#03, 14-26 v \#05, 12-28 v \#06 \\
\hline 15 Booot 6.3 .1 x64 1800X $3.6 \mathrm{GHz}$ & 3356 & $22 / 22$ & 960 & $51 \%$ & 3349 & 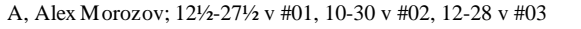 \\
\hline 16 Komodo 11.01 x64 Q6600 $2.4 \mathrm{GHz}$ & 3340 & $27 / 27$ & 642 & $50 \%$ & 3339 & $91 / 2-301 / 2 v \# 02,11^{1 / 2-281 / 2} \mathrm{v} \# 03,12^{1 / 2-271 / 2} \mathrm{v} \# 04,16-24 \mathrm{v} \# 05$ \\
\hline 17 Komodo 9.1 x64 Q6600 2.4 GHz & 3338 & $20 / 19$ & 475 & $72 \%$ & 3176 & 8-34 v \#03, 13-27 v \#06, 14-26 v \#09, 111⁄2-281/2 v \#11 \\
\hline 18 Stockfish 6 x64 Q6600 2.4 GHz & 3326 & $21 / 20$ & 256 & $69 \%$ & 3190 & $7-33 \# 03,14 \frac{1}{2}-251 / 2 v \# 09,11^{1 / 2-281 / 2} \vee \# 11,151 / 2-241 / 2 v \# 13$ \\
\hline 23 Arasan 21.2 x64 $1800 \times 3.6 \mathrm{GHz}$ & 3279 & $28 / 29$ & 600 & $38 \%$ & 3360 & A, Jon Dart; 51/2-341/2 v \#01, 5-35 v \#02, 101/2-291/2 v \#04 \\
\hline 24 Komodo 7 x64 Q6600 2.4 GHz & 3269 & $23 / 23$ & 974 & $65 \%$ & 3159 & $71 / 2-32^{1} / 2 v \# 03,11^{1 / 2}-281 / 2 v \# 09,10^{1 / 2-291 / 2} v \# 13,14-26 v \# 14$ \\
\hline 25 Arasan 21.2 x64 Q6600 $2.4 \mathrm{GHz}$ & 3251 & $44 / 46$ & 240 & $41 \%$ & 3312 & $4-36$ v \#02, 41ำ-351/2 v \#05, 7-33 v \#07, 23-17 v \#22 \\
\hline 26 Komodo 5.1 x64 Q6600 $2.4 \mathrm{GHz}$ & 3245 & $22 / 22$ & 038 & $64 \%$ & 3144 & 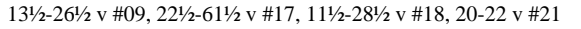 \\
\hline 27 Wasp 3.5 x64 1800X $3.6 \mathrm{GHz}$ & 3223 & $29 / 31$ & 600 & $32 \%$ & 3349 & A, John Stanback; 5-35 v \#02/03, 4ำ-351/2 v \#04, 7-33 v \#05 \\
\hline 28 Deep Hiarcs 14 1800X $3.6 \mathrm{GHz}$ & 3222 & $25 / 26$ & 760 & $39 \%$ & 3296 & A, Mark Uniacke; $7-33 \mathrm{v} \# 03,71 \frac{1}{2}-32^{1 / 2} \mathrm{v} \# 04,51 / 2-341 / 2 \mathrm{v} \# 06$ \\
\hline 29 Deep Rybka 4 x64 Q6600 2.4 GHz & 3198 & $19 / 19$ & 408 & $64 \%$ & 3098 & A, IM Vasil Rajlich; 51/2-341/2 v \#08, 91/2-301/2 v \#13, 8-32 v \#17 \\
\hline 30 Deep Hiarcs 14 Q6600 2.4 GHz & 3188 & $19 / 18$ & 450 & $61 \%$ & 3112 & 91/2-301/2 v\#13, 9-31 v \#14, 131/2-261/2 v\#16, 12-28 v \#17 \\
\hline 31 Chiron $3.01 \times 64 \mathrm{Q} 66002.4 \mathrm{GHz}$ & 3178 & $27 / 27$ & 656 & $45 \%$ & 3214 & A, Ubaldo Andrea Farina; 7-33 v \#09/13, 101/2-291/2 v \#14 \\
\hline 32 Wasp 3.5 x64 Q6600 $2.4 \mathrm{GHz}$ & 3170 & $38 / 39$ & 320 & $44 \%$ & 3216 & 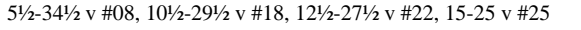 \\
\hline 33 Deep Junior Yokohama x64 Q6600 2.4 GHz & 3126 & $22 / 22$ & 010 & $42 \%$ & 3183 & A, Amir Ban \& Shay Bushinsky; 61/2-731/2v \#09, 71/2-721/2 v \#13 \\
\hline 34 Hiarcs 14 Athlon $1.2 \mathrm{GHz}$ & 3100 & $29 / 29$ & 560 & $55 \%$ & 3065 & $51 / 2-341 / 2 v \# 11,7-33$ v \#17, 91/2-301/2 v \#18, 7-33 v \#20 \\
\hline 35 Deep Fritz 13 Q6600 2.4 GHz & 3097 & $24 / 24$ & 826 & $55 \%$ & 3064 & A, Frans Morsch; 9-31 v \#24, 131/2-261/2 v \#26/29, 12-28 v \#30 \\
\hline 36 The Baron 3.43 x64 1800X $3.6 \mathrm{GHz}$ & 3088 & $29 / 31$ & 680 & $26 \%$ & 3272 & A, Richard Pijl; 21/2-371/2 v \#03, 31/2-361/2 v \#06, 3-37 v \#09 \\
\hline 37 Revelation 2 Hiarcs 14.1 PXA320 $800 \mathrm{MHz}$ & 2924 & $47 / 46$ & 220 & $55 \%$ & 2889 & A, Ruud Martin and Mark Uniacke; 7-13 v D Junior 12 Q6600 \\
\hline 47 Millennium ChessGenius Excl. M7 $300 \mathrm{MHz}$ & 2244 & $68 / 62$ & 120 & $62 \%$ & 2159 & A, Richard Lang; 61/2-131/2 v Rebel 9 P90 \\
\hline 48 Millennium ChessGenius Pro M4 120 MHz & 2165 & $59 / 54$ & 160 & $63 \%$ & 2068 & A, Richard Lang, 51/2-141/2 v Revelation Ruffian \\
\hline 49 Millennium ChessGenius ARM M4 $48 \mathrm{MHz}$ & 2075 & $51 / 47$ & 211 & $63 \%$ & 1986 & A, Richard Lang; 7-13 v MCG Pro, 8-12 v Montreux \\
\hline 50 Mephisto London $6800012 \mathrm{MHz}$ & 2006 & $59 / 58$ & 140 & $53 \%$ & 1983 & A, Richard Lang; $61 \frac{1}{2-1} 13^{1} / 2$ v MCG Pro, $10-10$ v MCG \\
\hline
\end{tabular}

Fig. 1. The recently tested 'Selected 50' from SSDF rating list '2020-07' of 2020-07-11, q.v., https://ssdf.bosjo.net. ${ }^{2,3,4}$

\footnotetext{
${ }^{1}$ Corresponding author, e'mail g.haworth@reading.ac.uk

2 'Games' $\equiv$ the number of games, played at ' $40 \mathrm{~m} / 2 \mathrm{hr}+20 \mathrm{~m} / 1 \mathrm{hr}$ ', on which the rating is based. 'Against' $\equiv$ average rating of opponents. '+' and '-' denote upper/lower 95\%-confidence intervals. 'A' $\equiv$ author(s), 'NE' $\equiv$ new entrant.

${ }^{3}$ Latest platform: AMD Ryzen 7 1800X, 8-core @ 3.6GHz, 16GB RAM, SSD, 6-man Syzygy EGTs.

${ }^{4}$ Fuller SSDF data including the '50' and long lists' match detail is available at http://centaur.reading.ac.uk/91758/.
} 